\title{
LA EDUCACIÓN POPULAR COMO PARADIGMA Y METODOLOGÍA DE ACCIÓN EN SALUD FAMILIAR
}

\author{
EDUCATION OF PUPULATION AS A PARADIGM AND \\ A METHODOLOGY OF ACTION IN FAMILY HEALTH
}

\author{
Luis Alberto Vivero Arriagada*
}

\begin{abstract}
RESUMEN
Objetivo: Reconocer y posicionar la educación popular como sustento paradigmático y metodológico para las acciones desarrolladas en el marco de la salud familiar. Metodología: Análisis de literatura desde una perspectiva hermenéutica crítica, que permite la comprensión de los aportes de los marcos teóricos a partir de una articulación con la experiencia práctica y académica de quien interpreta. Resultados: La salud familiar como modelo de acción reconoce en esta unidad y sus miembros su foco de acción, lo cual requiere necesariamente una resignificación no sólo de lo que ocurre en éste ámbito, sino también una lectura crítica de los paradigmas que sustenta las prácticas desde el sistema de salud y sus agentes. Conclusiones: Se plantea que la educación popular puede constituirse como un paradigma, y a la vez una propuesta metodológica, que ponga como centro de la acción el reconocimiento de la persona como un actor protagonista de su historia y sus prácticas, reconociendo sus saberes y cultura.
\end{abstract}

Palabras clave: Salud familiar, familia, educación.

\begin{abstract}
Objective: To recognize and position the education of the population as a paradigmatic and methodological basis for actions in the framework of family health. Methodology: Literature analysis from a critical hermeneutic perspective for an understanding of the contributions of different theoretical frameworks, based on coordination with the practical and academic experience of the interpreter. Results: Family health as an action model recognizes its focus of action in this unit and its components. This necessarily requires not only redefinition of what happens in this area, but also a critical reading, from the perspective of the health system and its agents, of the paradigms on which these practices are based. Conclusions: The paper proposes that education of population may constitute a paradigm, and simultaneously a methodological proposal, focusing on recognition of the person as protagonist in his own history and practices, while recognizing his knowledge and culture.
\end{abstract}

Key words: Family health, family, education.

Fecha recepción: 08/11/12 Fecha aceptación: 11/11/13

\footnotetext{
* Trabajador Social. Departamento de Trabajo Social, Universidad Católica de Temuco-Chile. Email: luisvive@gmail.com
} 


\section{INTRODUCCIÓN}

El desarrollo del presente trabajo se desprende desde un análisis y reflexión, que se sitúa desde la concepción de praxis, la cual se asume como una articulación permanente entre reflexión/acción, teoría/práctica, concreto/abstracto. Se plantea como objetivo el reconocer y posicionar la educación popular como sustento paradigmático y metodológico para las acciones desarrolladas en el marco de la salud familiar, particularmente un aporte teórico-práctico para las acciones socioeducativas de enfermería. En términos teleológicos, la perspectiva de educación popular, definida por Paulo Freire $(1,2)$, busca la construcción constante de nuevos saberes que contribuyan a la transformación social.

El abordaje metodológico en el cual se sustenta este trabajo se inscribe en una perspectiva de carácter hermenéutico-crítico, consecuente con la concepción de praxis que anteriormente se ha señalado. En tal sentido, la revisión documental que sirve de respaldo teórico conceptual se articula con la experiencia que tiene el autor de este ensayo, de más de 10 años de trabajo en el campo de la atención primaria de salud, articulado con su ejercicio académico universitario. Este círculo hermenéutico $(3,4)$ busca entregar elementos para la discusión en torno a una realidad contradictoria y conflictiva, que permita fundamentar la necesidad de resignificar y humanizar las acciones desplegadas por los agentes de salud desde las acciones desarrolladas en el marco de salud familiar, poniendo la educación popular como referente paradigmático y sustento metodológico de dicha acción. Estos elementos pueden ser de utilidad para el desempeño del rol educativo del/la enfermero/a, quien actúa como agente articulador entre los distintos profesionales de salud y los agentes comunitarios y sociofamiliares.

En este trabajo se entregan algunos elementos históricos generales, respecto de la emergencia de la salud familiar en América Latina, principalmente en virtud de los cambios epidemiológicos, sociodemográficos y los compromisos adoptados por los estados miembros de la Organización Mundial de la Salud y la Organización Panamericana de la Salud (OMS/OPS). Luego se articulan los cambios societales, que se expresan en ciertos indicadores epidemiológicos, con los cambios experimentados por la familia contemporánea, entendida ésta como una de las más significativas instituciones sociales. Finalmente se levanta una reflexión en forma de propuesta para el abordaje de los fenómenos sociofamiliares, desde una perspectiva de educación popular, reconociendo los saberes socioculturales propios de cada realidad histórica y cultural en que se sitúa una familia, como el sustento básico para la generación de cambios en ciertas prácticas que puedan repercutir negativamente en la salud de las personas o, por el contrario, fortalecer y validar aquellas que se inscriben en la línea de los factores condicionantes y protectores de la salud.

\section{Algunos antecedentes de la salud familiar en América Latina y el Caribe}

Al comenzar la década de los ochenta se había implementado la medicina familiar en tres países de América Latina (México, Panamá y Bolivia) con un total de 21 programas (5). Diez años después, en 1990, los países con medicina familiar eran 18: Argentina, Bolivia, Brasil, Colombia, Costa Rica, Cuba, Chile, República Dominicana, Ecuador, Jamaica, México, Nicaragua, Panamá, Paraguay, Perú, Puerto Rico, Uruguay y Venezuela, y reunían un total de 160 programas. Los primeros programas de medicina familiar se desarrollaron en México en la década del setenta, bajo el alero del Instituto Mexicano de los Seguros Sociales (IMSS), el cual recibe el reconocimiento por parte de la Universidad Nacional Autónoma de México (UNAM), creando en el año 1975 el Departamento de 
Medicina Familiar (5).

El caso de Cuba, que es reconocido como uno de los países de América Latina y el Caribe que más promueve un enfoque de salud familiar, sólo a partir de la década del ochenta incorpora esta perspectiva en sus diseños de salud pública, la cual se desarrolla en cuatro etapas (6): 1) fase política y de puesta en marcha (1984 hasta 1989), 2) fase de estancamiento de 1990 hasta junio 1994 (coincide con la caída del Muro de Berlín, 3) fase de perfeccionamiento metodológico (1994 hasta 2002) y 4) fase del Proyecto Revolución (2002 hasta la actualidad).

La década del noventa, a la luz de los cambios sociodemográficos y epidemiológicos experimentados en el último medio siglo, los programas de salud, bajo la orientación de la OMS y la OPS, ponen énfasis en la prevención y la promoción, a partir de fortalecer los determinantes de la salud, lo cual se manifiesta en una serie de conferencias y congresos realizados para abordar los nuevos fenómenos asociados a la salud $(5,7)$. En noviembre de 1994 se realizó en Canadá la Conferencia sobre Medicina Familiar en la Reforma de los Servicios de Salud de las Américas organizado por WONCA/OMS, desde la cual se desprende un documento aprobado por lo participantes con un título bastante sugerente: "Hacer que la práctica médica sea más relevante a las necesidades de salud de la comunidad" (5). En septiembre de 1996 tuvo lugar en Buenos Aires la conferencia regional de líderes y expertos de las Américas, sobre "La medicina familiar en la reforma de los servicios de salud" (5).

En el ámbito local, un estudio realizado por la Universidad Católica de Chile en el año 2011 (8) describe algunas problemáticas de la salud familiar en Chile y la falta de especialistas en el área de la salud familiar, destacando algunas opiniones de expertos, en las que señalan que existe "escasa valoración social del médico familiar, conflictos con otros especialistas respecto de los ámbitos de incumbencia". De acuerdo a lo planteado por algunos autores $(5,7,9)$, la salud familiar puede considerarse como un fenómeno complejo y multidimensional, que afecta al conjunto de los miembros de la familia, en término de su funcionamiento efectivo, en la dinámica interaccional, en la capacidad de enfrentar los cambios del medio social y del propio grupo, en el cumplimiento de las funciones para el desarrollo de sus integrantes propiciando el crecimiento y desarrollo individual, según las exigencias de cada etapa de la vida $(7,9,10)$.

Para Peñaloza et al. (11), en el enfoque de salud familiar, ésta adquiere un papel fundamental, pues constituye el ámbito de mayor influencia en la persona, a lo largo de toda su vida es la que le brinda cariño, seguridad, estabilidad, formación, cobijo, alimentación y otros elementos básicos para su desarrollo saludable. Por lo tanto el debilitamiento o falta de uno o varios de estos elementos puede ser factor causal o agravante de una enfermedad, por lo cual la familia constituye un campo de acción $(10,12)$ importante para la toma de decisiones para la salud y calidad de vida de sus componentes.

La OMS/ OPS toma como estrategia el enfoque de promoción de la salud, la cual queda expresada en el año 1997 con la Declaración de Yakarta (13), que viene a reforzar los acuerdos y compromisos asumidos por los países miembros de estas organizaciones, en cuanto a adoptar una estrategia mundial de salud para todos y observar los principios de atención primaria de salud establecidos en la Declaración de Alma-Ata de 1978 (7, 9). Con la Declaración de Yakarta se viene a reinstalar a la familia -y no la enfermedadcomo el centro de atención de las acciones de salud, abordando la estrategia de la Promoción de Salud, que permite ampliar más aún el campo de acción $(10,11)$.

El modelo de salud familiar presenta una serie de desafíos que se expresan tanto en la estructura del sistema de salud como en la praxis del modelo. Por un lado aquellos desafíos referidos a estrategias metodológicas y 
prácticas del abordaje de las problemáticas de salud que se expresan en la familia, sus componentes y sus relaciones sociocomunitarias, culturales e históricas, y por otro lado, en aspectos de carácter simbólicos (haciendo la distinción con las necesidades de tipo material y técnica), que demandan un cambio ideológico en la formación misma de los agentes de salud, en el sistema de salud y sus diferentes actores (12-14). Desafíos que van mucho más allá de las diferencias conceptuales entre un modelo biomédico respecto de uno de carácter bio-sicosocial.

\section{La educación popular como sustento epis- témico-metodológico de la salud familiar}

Las prácticas en el ámbito de la salud familiar que desarrollan los equipos de salud y en su interacción con otros agentes interpela a plantear un sustento paradigmático, que resignifique y valorice los distintos saberes y promueva relaciones horizontales y democráticas, tanto al interior de los equipos como en su relación con los actores externos al sistema de salud (14). En este sentido, se estima que la educación popular que nos propone Paulo Freire (13-15), cumple con esta premisa democratizadora y humanizante. Se plantea la educación popular como paradigma y metodología, por cuanto entrega elementos teóricos, epistemológicos y prácticos, que permiten comprender críticamente las instituciones y relaciones sociales y, a la vez, desarrollar una praxis que ponga como centro el reconocimiento del ser humano, como actor protagonista de su historia y sus prácticas $(2,15)$.

Se asume como premisa que en los equipos de salud predomina un saber y una práctica fundamentada en una racionalidad técnicocientífica (racionalista/empirista) (16), expresado concretamente en el uso hegemónico del método científico. Por lo anterior, no resulta fácil promover una práctica en el campo de la salud familiar que tenga un fundamento axiológico y metodológico contrario a esta lógica positivista, lo cual implica un desafío importante para los actores involucrados. La hegemonía del saber científico y técnico (16), fundamentado en la concepción positivista, lleva a que las relaciones al interior del sistema de salud se den sobre la base de prácticas verticales, de acuerdo a segmentos con niveles diferenciados de poder (17) y capacidad resolutiva, e incluso con formas autoritarias en la configuración de ciertas relaciones cotidianas, que limita o impide que los sujetos y sus familias puedan ser agentes de su propia recuperación o, en palabras de Freire, de su emancipación (1).

Por ello es que se plantea desafiar el saber científico racional-instrumental $(16,18)$, a partir de una praxis pedagógica humanista y democrática, fundamentada en los planteamientos de Paulo Freire $(1,2,15)$. A partir de una profunda y permanente reflexión crítica y humanista, se promueve un reconocimiento de todos los saberes, a una democratización profunda del conocimiento y no instrumentalizarlo a los intereses de dominación de la clase dirigente (2). Esto implica un giro - o a lo menos un desplazamiento- epistemológico y político en las estructuras y prácticas rígidas, verticales e incluso autoritarias que se generan en el campo de salud.

Las acciones de salud, desarrolladas en las relaciones cotidianas con las personas y sus familias, deben sustentarse en una praxis liberadora, entendida como reflexión y acción de los hombres y las mujeres sobre el mundo para su transformación (2). Sin una práctica crítica, democrática y humanizante, es imposible la superación de la contradicción opresor/oprimido $(1,2)$. Pero en ciertos casos, pareciera que las formas educativas que se canalizan desde los equipos de salud parten asumiendo, como único saber válido, aquel que está respaldado por el conocimiento científico y técnico, en cuanto sustento ideológico de su acción y su poder (14, $16,19,20)$. Si no se considera, no se reconoce ni respeta la visión de esos otros saberes, las acciones educativas en el ámbito de la salud 
familiar, se constituye en una especie de invasión cultural, realizada quizás con la mejor de las intenciones, pero invasión cultural al fin $(14,15,20)$.

En estas condiciones, las acciones de educación que se operacionalizan desde los equipos de salud, liderados generalmente por los y las profesionales de ésta área, se asemejan mucho a lo que Freire (2) denomina educación bancaria, en tanto acción en la cual el educador deposita los contenidos en el educando. Por ello es que se propone que los actores deben generar nuevas prácticas de interacción en sus relaciones con las personas a nivel individual, familiar y colectivo, orientadas la humanización y liberación de los hombres y mujeres en permanente interacción y respeto.

La salud familiar no puede ser sólo una herramienta accesoria e instrumental en las acciones de los equipos multidisciplinarios de salud, sino que debe contribuir en proponer e instalar enfoques teóricos y metodológicos en lo que se refiere a los niveles de trabajo con familias y la comunidad, por ejemplo en lo que respecta a la educación permanente en salud.

\section{CONSIDERACIONES FINALES}

Se propone que los actores de salud generen nuevas prácticas de interacción en sus relaciones con las personas a nivel individual, familiar y colectivo, que contribuya a superar la contradicción oprimido/opresor (2). Por lo tanto, es necesario avanzar en la superación de la lógica autoritaria que implica situar el saber científico-técnico como el único y verdadero, en desmedro del conocimiento popular.

Es necesario instalar la reflexión y análisis crítico - un proceso de conciencia críticaque implique una reflexión crítica, democrática, humanista e intercultural, al interior de los propios equipos de salud, respecto del hacer de cada actor de los equipos y el hacer de estos grupos de trabajo inter-multidisciplinario, y en aquello referido a la acción desarrollada con otros actores, en los diferentes espacios socioculturales en los que se interactúa. Se requiere una relectura que permita una nueva interpretación, comprensión y valoración de los saberes de la vida colectiva, familiar e individual, aprender de ello e integrarlos como nuevos saberes en las acciones de salud.

La educación popular en el ámbito de un modelo de salud familiar posibilita una nueva praxis, que implica la reflexión en torno a la participación de los distintos actores (de la salud y las familias) sobre su propio ser, de sus potencialidades, de sus saberes y en el desarrollo de ese poder en la explicación y comprensión de sus potencialidades (material y simbólico), de la cual nacería su capacidad de opción de sus distintos haceres en la vida cotidiana y por cierto, en aquellas referidas a su salud en el más amplio sentido.

La educación popular freiriana se sustenta sobre una sólida base epistemológica y política, que permite la construcción de un saber dialéctico y educativo, desde la realidad latinoamericana, una problematización e interpretación en y desde las prácticas sociales cotidianas, en este caso expresadas en lo que respecta a la salud de las familias y sus integrantes, unidos en una permanente construcción histórica y cultural, en donde los profesionales de la salud son uno más de este componentes en interacción y diálogo democrático.

\section{REFERENCIAS}

1. Freire P. La educación como práctica de la libertad. Argentina: Siglo XXI; 2004.

2. Freire P. Pedagogía del oprimido. Argentina: Siglo XXI; 2006.

3. Echeverría R. El Búho de Minerva. Chile: Dolmen Ediciones; 1997. 
4. Giddens A. Las nuevas reglas del método sociológico. Argentina: Amorrortu Ediciones; 1998.

5. Ceitlin J. La medicina familiar en América Latina. Atención Primaria 2006; 38(9): 511-514.

6. Sansó F. Veinte años del modelo cubano de medicina familiar. Revista Cubana de Salud Pública [Internet]. 2005 [citado 22 jul 2012]; 13(2). Disponible en: http:// scielo.sld.cu

7. Arriagada I. Familia y políticas públicas en América Latina: Una historia de desencuentros. Santiago, Chile: CEPAL; 2007. 178 p.

8. Rojas MP, Judith S, Erazo Á, Klaus P. VI Concurso políticas públicas. Propuestas para Chile. Pontificia Universidad Católica de Chile; 2011.

9. Cerrutti M, Binstock G. Familias latinoamericana en transformación: desafíos y demandas para la acción pública. Serie políticas sociales No 147. CEPAL. 2009; $61 \mathrm{p}$.

10. Giddens A. Un mundo desbocado .Los desafíos de la globalización en nuestras vidas. Argentina: Taurus; 2003.

11. Peñaloza B, Rojas MP, Rojas P, Téllez A. Modelo de atención en salud familiar. Una propuesta para contribuir al cambio [Internet]. Programa de Medicina
Familiar, Pontificia Universidad Católica de Chile, Santiago; 2001 [citado 22 jul 2012]. Disponible en: http://www.medicina-familiar.cl/sitio/images/stories/familiar.pdf

12. Bourdieu P. Capital cultural, escuela y espacio social. 7a. ed. México, D.F: Siglo Veintiuno editores; 2007.

13. Declaración de Yakarta sobre promoción de salud en el siglo XXI. OMS/OPS; 1997.

14 Vivero L. La participación social en salud a partir del discurso de los profesionales médicos dependientes de Servicio de Salud Valdivia [Tesis Maestría]. [Temuco, Chile]: Universidad de la Frontera; 2005.

15. Freire P. Pedagogía de la esperanza. Un reencuentro con la Pedagogía del Oprimido. Argentina: Siglo XXI; 2002.

16. Habermas J. Ciencia y técnica como ideología. Madrid: Editorial Tecnos; 1999.

17. Foucault M. Microfísica del poder. Madrid: La Piqueta; 1992.

18. Mc Carthy T. La Teoría crítica de Jurgen Habermas. Madrid: Editorial Tecnos; 1978.

19. Foucault M. La vida de los hombres infames. Argentina: Editorial Altamira; 1996.

20. Vivero L. Participación social en salud y conocimiento popular: ¿realidad o retórica política? Revista Regional de Trabajo Social, No 40, Uruguay. 2007. 